This is an author produced version of a paper published in Biochimica et Biophysica Acta. This paper has been peer-reviewed but does not include the final publisher proof-corrections or journal pagination.

Citation for the published paper:

Duan, RD.

"Alkaline sphingomyelinase: An old enzyme with novel implications" Biochimica et Biophysica Acta, 2006, Vol: 1761, Issue: 3, pp. 281-91. http://dx.doi.org/10.1016/j.bbalip.2006.03.007ea

Access to the published version may require journal subscription. Published with permission from: Elsevier 


\title{
Alkaline sphingomyelinase: an old enzyme with novel implications
}

\author{
Rui-Dong Duan
}

Gastroenterology Lab, Biomedical Center B11, Lund University, S-221 84 Lund, Sweden

Address for correspondence:

Rui-Dong Duan, MD, PhD,

Gastroenterology Lab,

Biomedical Center, B11,

S-221 84 Lund,

Sweden

Tel. +46462220709

Fax. +4646137277

Email: Rui-dong.duan@med.lu.se 


\section{Summary}

Alkaline sphingomyelinase (alk-SMase) is present in the intestinal tract and additionally human bile. It hydrolyses sphingomyelin in both intestinal lumen and the mucosal membrane in a specific bile salt dependent manner. The enzyme was discovered 36 years ago but got real attention only in the last decade, when sphingomyelin metabolism was realized to be a source of multiple lipid messengers, and when dietary sphingomyelin was found to inhibit colonic tumorigenesis in animals. The enzyme shares no structural similarity with other SMases and belongs to the nucleotide pyrophosphatase/phosphodiesterase family. The enzyme is of specific properties, such as bile salt dependency, trypsin resistance, high stability, and tissue specific expression. In the colon, the enzyme may play antiproliferative and antiinflammatory roles through generating ceramide, reducing the formation of lysophosphatidic acid, and inactivating platelet-activating factor. The enzyme is down regulated in human long-standing ulcerative colitis and colonic adenocarcinoma, and mutation of the enzyme has been found in colon cancer cells. In the small intestine, alk-SMase is the key enzyme for sphingomyelin digestion. The hydrolysis of sphingomyelin may affect the cholesterol uptake and have impact on sphingomyelin levels in plasma lipoproteins. The review summarizes the new information of alk-SMase from biochemical, cell and molecular biological studies in the last decade and evaluates its potential implications in development of colon cancer, inflammatory bowel diseases, and atherosclerosis. 


\section{Historical aspects of alkaline sphingomyelinase.}

Alkaline sphingomyelinase (alk-SMase) is an old enzyme, which was first identified in the intestinal tract by Nilsson in 1969 [1], 3 years after the discovery of acid SMase [2, 3], and 7 years before the discovery of neutral SMase [4] in mammalian tissues. In contrast to the rapid research progresses made for both acid and neutral SMases soon after their discoveries, not a single study on alk-SMase had been reported until the middle of nineties. This can probably be attributed to several factors. The discovery that acid SMase deficiency is the genetic cause for Niemann Pick disease [5] stimulated the research on acid SMase, whereas the co-localization of neutral SMase, sphingomyelin (SM), and cholesterol in the cell membrane attracted scientists to study neutral SMase and its effects on membrane properties. Alk-SMase, although discovered in the early era, was only considered to be involved in digestion of SM, which was at that time not considered to be an important nutritional component, and whose signalling roles had not been identified. In the mid nineties we started to investigate alk-SMase mainly for two reasons. The first was the insight that metabolism of SM generates antiproliferative messengers, such as ceramide and sphingosine, and proliferative messengers, such as sphingosine-1-phosphate, ceramide phosphate, and lysosphingomyelin. The biological actions and the pathways affected by these compounds have been intensively reviewed [6-10]. The second reason was the finding that supplementary SM in the diet inhibits the formation of colonic aberrant crypt foci and colonic tumours caused by chemical carcinogens in animal studies [11]. Thus in 1995, 26 years after its discovery, the enzyme got attention again and was named alk-SMase [12]. One year later, the enzyme activity was also found in human bile but not in bile of other 
species [13, 14]. The alk-SMase in human bile was purified in 1997 [15] and those in rat and human intestinal tract were purified in 2002 and 2003, respectively [16, 17]. The enzyme is now cloned and the human gene is found to be located at chromosome $17 \mathrm{q} 25$ $[18,19]$. The reduction of the enzyme activity in human colon cancer was reported in 1997 [20] and the first mutation of the enzyme was discovered in 2004 in one human colon cancer cell line [21]. The major events related to the enzyme are listed in Table 1.

\section{Alk-SMase, a novel member of NPP family.}

Although alk-SMase was originally identified as a SMase that catalyses the breakdown of SM by a phospholipase $\mathrm{C}$ activity, gene cloning shows that the sequence of alk-SMase shares no similarities with either acid or neutral SMase but about 30\% similarities with the members of the nucleotide pyrophosphatase/phosphodiesterase (NPP) family [18]. Being a novel member of the NPP family, the enzyme is also called NPP7. The members in the NPP family are characterized as ecto-enzymes, which bind the plasma membrane via either $\mathrm{N}$ or $\mathrm{C}$ terminal transmembrane domain, with the rest part of the enzyme including the catalytic domain located outside the cells. The structural and functional diversities of NPP members have been recently reviewed [22].

The structure of alk-SMase is illustrated in Fig. 1. The enzyme has a hydrophobic domain at both the $\mathrm{N}$ and the $\mathrm{C}$ terminals. The one at the $\mathrm{N}$ terminal is a signal peptide, which is cleaved in the mature enzyme, and that at the C-terminal is a signal anchor, which hooks the enzyme on the plasma membrane. Expression of the enzyme lacking the signal anchor in cultured cells increases the enzyme levels in the medium [23]. Immunogold labelling showed that the enzyme is located at the surface of microvilli 
membrane in the intestinal tract [17]. At the upstream of the signal anchor there is a tryptic site that is cleaved by pancreatic trypsin, resulting in release of the enzyme into the intestinal lumen [23]. The enzyme can also be dissociated from the mucosal membrane by bile salts with probably a non-specific detergent effect [24]. Both the membrane bound form and the secreted form are active, and the latter induced by pancreatic trypsin has higher activity than the membrane bound form [23]. As shown in figure 1 , the enzyme has $5 \mathrm{~N}$-glycosylation sites, which are all glycosylated in the mature enzyme. Only the fully glycosylated enzyme is transported to the plasma membrane and fully active. The non-glycosylated and partly glycosylated enzymes are trapped in intracellular compartments with little activity. Mutation of the N-glycosylation sites markedly reduced the enzyme activity [25]. The transport of the enzyme to the membrane is rapid, as our unpublished data show that the levels of the enzyme in the intestinal mucosa can be replenished within 10 min after depletion of the enzyme by high concentrations of trypsin. Similarly rapid transportation from intracellular pools to plasma membrane in basophils during allergic reaction was also reported for NPP3, an NPP member that is also expressed in the brush border of enterocytes [26, 27]. Inside the cells, alk-SMase was found in the endosome-like structures near plasma membrane [17], which may indicate that the enzyme is transported in the endosomes to the plasma membrane, or alternatively internalised and trapped in the endosomes.

Apart from the glycosylation sites, alk-SMase has 6 amino acid residues that form two predicted metal coordinating sites (Fig 1), which are conserved in all NPP members. These metal coordinating sites are believed to be important for metal (mainly $\mathrm{Zn}^{2+}$ ) dependent activation of the NPPs [28]. But in contrast to most NPP family members, the 
activity of alk-SMase against SM under optimal conditions is not stimulated by $\mathrm{Ca}^{2+}$ and $\mathrm{Mg}^{2+}$, but inhibited by $\mathrm{Zn}^{2+}[18]$. However, by mechanisms not understood, mutation of any amino acid residue that forms the metal coordinating sites abolishes alk-SMase activity against SM [25], indicating still a crucial role of these sites in the enzyme function. Also shown in Fig.1, the predicted catalytic sites, the glycosylation sites, and metal coordinating sites are formed by amino acids encoded by codons distributed in different exons. Mutations of any amino acid forming these sties often cause significant reduction of the enzyme activity [25]. The activity of the enzyme is therefore not critically determined by a single domain but by the integrity of the whole molecule.

\section{Unique Properties}

Differing from other NPPs and acid and neutral SMases that are expressed widely in many types of cells [29], expression of alk-SMase is so far confined to the intestinal mucosa in many species except guinea pig, and additionally to human bile, but not the bile of other species including baboon, pig, rat, mouse, rabbit, and guinea pig [14]. Western blot identified the protein only in the intestinal mucosa but not in other organs, including brain, heart, lung, liver, spleen, pancreas, kidney, and skeletal muscle in rats [16]. Northern blot showed high levels of the enzyme mRNA only in the intestine and additionally in human liver, but not rat liver $[18,19]$. The enzyme in human liver is likely localized in the canalicular membrane, dissociated by bile acid, and secreted in the bile. In the intestinal tract, the activity is low in the duodenum and colon and high in the jejunum. Phenogenetic tree generated from either Swiss-Prot [18] or Genetyx [30] shows that the evolution of alk-SMase is a late event, and the enzyme represents a new 
branch in NPP family, which probably accounts for the finding that the activity is present in the intestine of chicken but not fish (unpublished data).

Besides the tissue-specific expression, alk-SMase has at least two other unique properties in comparison with other NPPs or SMases. The first is the activity dependency on bile salts. In the absence of bile salt, the activity of alk-SMase is very low, and it increases with the increasing monomeric concentrations of bile salts; the maximal activation occurs at the critical micelle concentrations (CMC) of each bile salt. Although all bile salts have stimulatory effect on the enzyme, the degree of the activation varies greatly among bile salt species. Taurocholate (TC) and taurochenodeoxycholate (TCDC), two salts of primary bile acids, are the most effective ones, which are about 20 fold more potent than other bile salts [16]. Other detergents, such as CHAPS and Triton X100 that are commonly used in acid and neutral SMase assay, do not stimulate rather strongly inhibit bile salt induced activation of alk-SMase. The mechanism of TC- and TCDCinduced activation is thus not related simply to detergent effects of the bile salts on the amphiphilic SM, but to a specific biologic interaction between these bile salts and the enzyme. Such a property is of physiological relevance, as alk-SMase so far has been found only in the places where bile salts are present. Alk-SMase is therefore the only SMase for which a physiological activator was identified. The second unique property of alk-SMase is the resistance to trypsin digestion. Unlike acid and neutral SMases in the intestinal tract that are rapidly inactivated by pancreatic trypsin [12], alk-SMase is stable when exposed to trypsin at a concentration as high as $1 \mathrm{mg} / \mathrm{ml}$. As already mentioned, trypsin indeed specifically releases alk-SMase from the intestinal mucosal and meanwhile 
increases its activity [23]. Thus alk-SMase is the main SMase that can well survive and be transported along the whole intestinal tract and found in the faeces.

\section{Functional implications in digestion of dietary SM.}

SM is a dietary component, being particularly abundant in milk, egg and meat [31, 32]. In the milk, SM accounts for about $20-35 \%$ of the total phospholipids and is present in the outer leaflet membrane of the milk fat globules [33]. In the intestinal tract, hydrolysis of dietary SM mainly occurs in the mid part of jejunum, where alk-SMase is abundant, indicating that the enzyme is responsible for SM digestion [34]. Although acid SMase activity is present in the intestinal tract, it is high in the duodenum but low in the other parts of the intestine [12]. Human milk contains acid SMase activity [35], but whether this activity plays a fundamental role in hydrolysing milk SM in the stomach is not clear. Analysis of SM breakdown products in different parts of the gastrointestinal tract in the new born after feeding milk show a marked formation of ceramide in the jejunum and ileum, but not in the stomach and duodenum, arguing against an important digestion of SM in the upper gastrointestinal tract [36]. The predicted process and regulation of SM digestion in human intestinal tract is shown in Fig.2. After a Western meal containing SM and other nutritional components, such as fat and proteins, cholecystokinin (CCK) will be released from endocrine cells in the intestine. The released CCK will stimulate the contraction of gallbladder and release alk-SMase as well as its natural activator bile salt in the bile to the intestinal lumen. Meanwhile, CCK will stimulate the secretion of trypsin from the pancreas, which in turn cleaves the alk-SMase bound on the intestinal mucosa, releases it into the intestinal lumen and enhances its 
activity [23]. Alk-SMase derived from the intestinal sloughing cells will also participate in the process. The formed ceramide will be further hydrolysed to sphingosine by a neutral ceramidase, which is also present in the intestinal tract in parallel with alk-SMase $[1,37]$. The intestinal neutral ceramidase has been recently purified and characterised [38]. The enzyme is a type 2 ceramidase, and shares high similarities to the ones identified in rat and mouse kidneys [39, 40]. Like alk-SMase, the intestinal neutral ceramidase is also a brush border enzyme, resistant to trypsin digestion, and activated by bile salts around the CMC [38]. Alk-SMase and neutral ceramidase act therefore concertedly in SM digestion in the gut. Studies from neutral ceramidase knockout mice recently confirmed the roles of the enzyme in hydrolysis of ceramide in the gut [41]. The formed sphingosine is absorbed into the enterocytes by a passive diffusion mechanism and converted to either ceramide or fatty acid in the enterocytes [42]. Since the sphingosine absorption can be inhibited by SM present in the mixed micelles [43], hydrolysis of SM by alk-SMase may enhance the absorption of sphingosine.

The alk-SMase in rat small intestinal content has the capacity to hydrolyse 8 $\mu$ mole SM in $1 \mathrm{~h}$ in the test tubes [23]. However, in vivo study showed that the animal is only able to completely digest less than $7 \mu$ mole SM in $24 \mathrm{~h}$ [34]. The limitation is probably caused by two factors. The first is that the presence of cholesterol, other phospholipids, and fat in the gut inhibits alk-SMase activity, as has been demonstrated both in vitro [44, 45] and in vivo [46]. The inhibition caused by cholesterol is likely related to the formation of SM/cholesterol complex due to the strong interaction of these two molecules [47], and the inhibition caused by other phospholipids is due to a competition of these lipids with SM for the substrate binding site of the enzyme [15]. The 
second factor that limits alk-SMase capacity is the high concentration of bile salts in the intestinal lumen. Although bile salts, particularly TC and TCDC, are required for activation of alk-SMase, the maximal stimulatory effects of bile salts occur at the CMCs. When the bile salt concentrations are higher than CMC, the stimulatory effects on both alk-SMase and neutral ceramidase decrease [16, 38]. Under physiological conditions, the concentration of bile salts in the upper part of small intestine is higher than their CMC. It is thus understandable why SM digestion occurs most effectively in the low part of the small intestine, where most fat and phospholipids have been digested and bile salt concentration has been reduced due to the absorption. Studies on the NPP family members showed that the phosphorylated products after substrate hydrolysis can bind the NPP enzyme with an affinity higher than the substrate, and prevent complete hydrolysis of the substrates $[29,48]$. Such a product-induced inhibition may not occur for alkSMase, as phosphocholine, the hydrolytic product of SM, does not inhibit but slightly increases alk-SMase activity against SM, at least in test tubes [44]. Owing to the slow and incomplete SM hydrolysis in the gut and the high stability of alk-SMase and neutral ceramidase [49], colon is therefore able to expose to undigested SM, unabsorbed ceramide, and also alk-SMase and neutral ceramidase from the upper small intestine.

\section{Functional implications in colon cancer and intestinal inflammation.}

In the last 10 years, an attractive finding in the SM field is a potential link between SM digestion and colon cancer. Investigators in Merrill's group have performed a series of experiments showing that supplementation of both natural and synthetic SM in mice can inhibit the chemical carcinogen induced formation of aberrant crypt foci in a 
short term and the development of colon cancer in a long term [11, 50, 51]. Because such preventive effects caused by SM can be reproduced by feeding ceramide or other sphingolipids that generate ceramide after hydrolysis, it is reasonable to believe that SM needs to be first hydrolysed for its anticancer effects. Alk-SMase, the key enzyme in the intestinal tract becomes naturally a topic for investigation. Hertervig et al determined alkSMase activity in the biopsy samples taken from human sporadic colonic adenomas, colorectal carcinomas, and the tissues of familial adenomatous polyposis, and found that the activity is decreased progressively from adenoma to carcinoma comparing with the adjacent tissues or normal tissues [20,52]. Not only in the biopsy samples, a marked reduction of alk-SMase activity in the stools of colorectal cancer patients compared with normal individuals was also recently reported [53]. The 70 to $90 \%$ reduction of alkSMase activity found in the colonic mucosa and content in colon cancer patients could reduce the hydrolysis of SM and the generation of ceramide. Indeed, accumulation of SM and reduction of ceramide formation in colon cancer have been reported [54, 55]. Ceramide, by activating specific phosphatases and proteases, can induce dephosphorylation of many proliferative and antiapoptotic molecules such as Bcl-2, Akt, and PKC $\alpha$, and can activate $\mathrm{Rb}$ and facilitate cytochrome $\mathrm{C}$ release from the mitochondria, leading to inhibition of cell proliferation, promotion of differentiation, and induction of apoptosis [7-10]. In addition, the generation of ceramide provides the substrate for ceramidase to form sphingosine, which is another antiproliferative and apoptotic molecule [7, 9], and may also contribute the anticancer effects of alk-SMase in the gut. In agreement with the concept, feeding milk SM in artificially reared rats 
stimulates crypt cell differentiation and increases the number of the mature epithelial cells at the tips of the microvilli [56].

Apart from hydrolysis of SM to generate ceramide, alk-SMase can also hydrolyse lyso-phosphatidylcholine (lyso-PC) with a phospholipase C activity and turn lyso-PC to monoacylglycerol [18]. In the intestinal tract, lyso-PC and SM are co-localised mainly at the apical part of brush border [57], where alk-SMase is abundant [17]. Lyso-PC is generally considered to be mitogenic. Under the actions of phospholipase D, lyso-PC will be hydrolysed to lysophosphatidic acid (LPA), which strongly stimulates the activities of Akt and Erk1/2, and the release of interleukin-8 through different types of $G$ protein coupled receptors, leading to cell proliferation and inflammation [58-60]. Recent studies indicate that LPA may have important roles in promoting colon cancer development and metastasis. The expression of LPA receptors in colon cancer cells may be upregulated [61]. In DLD1 cells, LPA was found to markedly increase cell migration and secretion of vascular endothelial growth factor even at nanomolar concentrations [62]. Alk-SMase, by cleaving off phosphocholine headgroup from lyso-PC, may reduce the formation of LPA and protect the cells from tumorigenesis. In the NPP family, NPP 2 also has activity against lyso-PC, but the activity is a type of phospholipase D not phospholipase C. NPP2 is therefore a potent mitogenic factor [63]. Within NPP family, alk-SMase is so far the only member that has anticancer properties.

It is well known that cancer often develops following chronic inflammations, and longstanding ulcerative colitis is a risk factor for colon cancer. The implication of alkSMase in inflammatory bowel disease was first indicated by a finding that longstanding ulcerative colitis was associated with about 25\% reduction of alk-SMase activity [64]. A 
recent finding cast a light on the mechanism for the potential effects of alk-SMase. As reported by Wu et al, alk-SMase could hydrolyse and inactivate platelet-activating factor (PAF) by a phospholipase C activity [65]. PAF is a type of phospholipids with an acetyl bond at the $\mathrm{C} 2$ and an ether bond at the $\mathrm{C} 1$ linking to an alkyl chain. PAF can be formed in the colonic mucosa and released into the lumen. Studies from different lines show that PAF is a potent proinflammatory factor that stimulates release of cytokines, chemotaxis, and cell proliferation, by activation of MAP kinase, NF-אB, PLA2, and phospholipase D [66, 67]. High levels of PAF have been found in the tissues of ulcerative colitis, necrotizing enterocolitis, gastrointestinal damage and colorectal cancer [68-70]. Increased PAF levels in the stools of human ulcerative colitis, Crohn's disease, and neonatal necrotizing enterocolitis have also been reported [71, 72]. The study by Wu et al [65] showed that alk-SMase cleaves the phosphocholine moiety from PAF and significantly inhibits PAF-induced MAPK activation and cytokine release from colon cancer cells, and blocked the chemotaxis in HL-60 cells. Other SMases have not been found to have activity against PAF. A neutral SMase was previously reported to be able to hydrolyse lyso-PAF but not PAF [73]. Due to the proinflammatory effects of PAF, efforts were previously made to develop drugs to inhibit PAF activity by inducing deacylation at the C2 position. Such efforts have not resulted in great success. The finding that alk-SMase inactivates PAF by attacking the C3 position may cast a light in the drug discovery area. In conclusion, alk-SMase may potentially inhibit colonic tumorigenesis and inflammation with three arms: generating ceramide, reducing LPA formation, and inactivating PAF, as shown in Fig. 3. Considering the potential roles of alk-SMase discussed above, the reduction of alk-SMase in colorectal cancer may render the 
enterocytes more susceptible to carcinogens and proinflammatory factors, and thus increase the risk of colon cancer.

The mechanism underlying the significant reduction of alk-SMase in colon cancers is not fully understood; inactivating mutation may be one factor underlying. As shown in Fig. 4, the wild type mRNA of alk-SMase has 5 translated exons, whereas in HT29 colon cancer cells, the exon 4 is deleted and 7 foreign amino acid residues are inserted due to alternative splicing [21]. A sole deletion of exon 4 without an insert of foreign amino acids was also found in a human colon cancer sample (unpublished data). Both mutations inactivate the enzyme activity. In the domain coded by exon 4 , there is a His residue that participates the formation of one of the two metal coordinating sites. Site substitution of the His residue resulted in loss of alk-SMase activity [21]. As to alkSMase in the liver, a short form that is composed of only exon 1 and 5 was identified in Hep G2 liver cancer cells [74]. In addition, by searching GenBank we found a cDNA (accession number 126250) that encodes the full-length alk-SMase plus a 73 amino acid tag at the N-terminal. The cDNA is derived from a liver tumour tissue. Our preliminary study showed that the addition of the tag markedly reduced the enzyme activity.

\section{Functional implications in cholesterol absorption and atherogenesis.}

Alk-SMase in the small intestine may affect cholesterol absorption. Previous studies showed that treating Caco-2 cells with bacterial SMase to decrease the membrane SM levels by $60 \%$ resulted in $50 \%$ reduction of cholesterol uptake, accompanied by a decrease in cholesterol synthesis [75]. Such inhibitory effects of SMase on cholesterol uptake were also demonstrated in rabbit proximal tubular cells of kidney [76]. Alk- 
SMase is the major SMase in the small intestinal tract. It is able to reduce the membrane SM levels in enterocytes to similar degree [77] and is thus expected to have similar effects on cholesterol uptake. On the other hand, as first reported by Nyberg et al [46], feeding animals with dietary SM significantly decreased cholesterol absorption in the intestine; the maximal inhibition occurs at an equal molar amount of SM and cholesterol. Milk SM was found to be more potent than egg SM, indicating that the inhibition is related to the degree of the saturation and the length of SM $[78,79]$.

The mechanism underlying the inhibitory effect of dietary SM on cholesterol absorption was previously thought to be related to the strong interaction of SM and cholesterol, because in mixed micelle model, SM reduces the thermodynamic activity of cholesterol significantly and the reductions correlate with the reduced uptake of cholesterol by Caco-2 cells [80]. Of interest is a recent finding that sphingosine can form condensed lipid complexes with the $\alpha$-face of cholesterol and inhibit cholesterol absorption [81]. The sphingosine-induced inhibition on cholesterol absorption was stronger in Caco-2 cells than in HT29 cells. Because Caco-2 cells express higher levels of Niemann-Pick C1 like 1 (NPC1L1) protein, the main intestinal cholesterol transporter [82], than HT29 cells, the involvement of NPC1L1 is indicated. However, the differential responses of Caco-2 and HT29 cells may also be related to alk-SMase, as Caco-2 cells express wild type alk-SMase whereas HT29 cells expressed an inactively mutant alkSMase [21]. The formation of sphingosine in the gut is a consequence of a concerted action of alk-SMase and neutral ceramidase. Alk-SMase that triggers the process of sphingosine formation may play important roles in such an inhibition. 
SM in the plasma is recently thought to have impact on the pathogenesis of atherosclerosis based on research findings from several lines. First, SM is present in different types of lipoproteins, being high in LDL. The arteriole has SMase that can hydrolyse SM in LDL to ceramide and induce lipoprotein aggregation [83, 84]. Delivery of the plasma SM and its hydrolytic products to arterial intima may affect development of atherosclerosis. Second, high levels of SM were present in the lesion of atherosclerosis [85-88] and in the plasma of several hyperlipidemic and highly atherogenic conditions, such as type II hyperlipidemia, diabetes, cholesterol fed rabbit, the apo E -/- mice, and high fat intake humans [89-92]. Finally, plasma phospholipid transfer protein that is involved in the phospholipid metabolism in HDL and apoB containing lipoproteins was found to be a risk factor for atherogenesis [93, 94]. However, whether SM digestion in the gut affects plasma SM levels in lipoproteins remains elusive. High SM diet was found to decrease lymphatic output of SM [78] but increase SM in non-HDL lipoproteins [95]. It would be important to identify the mechanism leading to the reduced lymphatic output and increased incorporation of SM into lipoproteins in these conditions. In both cases, alk-SMase, the key enzyme responsible for hydrolysis of SM in the gut could play important roles.

\section{Regulation of alk-SMase}

The expression of alk-SMase is changed in response to dietary factors. In animal studies, high fat diet (53\% energy) was found to greatly reduce the alk-SMase activities in the intestinal mucosa by $50 \%$. The reduced activity is caused by an inhibited expression of the enzyme as shown by Western blot [96]. However, whether the 
inhibitory effects are mediated by free fatty acids has not been studied in detail. What we know now is that neither arachidonic acid [97] nor fish oil (unpublished data) in the diet affect alk-SMase activity in rat colon. On the other hand, certain types of dietary fiber affect the expression of alk-SMase. We previously showed that administration of $10 \%$ water soluble fiber psyllium [96], but not the water insoluble fiber cellulose [98] increased both the activities and protein of alk-SMase in the colon of mice [96]. The opposite effects of fat and fiber on alk-SMase expression may have relevance to the different effects of these two dietary factors on colon cancer development.

Apart from the dietary factors, two chemical agents have been shown to increase alk-SMase levels. The first is ursodeoxycholic acid (UDCA). Administration of UDCA for 10 days increased the colonic alk-SMase activity in rat colon in a dose-dependent manner [99]. Recent studies on Caco2 cells cultured under both monolayer and polarized conditions indicate that UDCA only increases alk-SMase activity in the absorptive epithelial cells not the crypt cells [100]. The second chemical agent that increases alkSMase is 5-aminosalicylic acid (5-ASA), an anti-inflammatory drug that can inhibit NF$\kappa \mathrm{B}$ and MAPK activities [101]. Feeding rats with 5-ASA for 10 days selectively increased alk-SMase in the colonic mucosa [102]. It is worth noting that both UDCA and 5-ASA have chemopreventive effects against colon cancer [103, 104].

\section{Problems and Future targets.}

Although the accumulated data indicate that alk-SMase may affect both physiological processes, such as maturation of intestinal tract, digestion of dietary SM, and cholesterol absorption, and pathological processes, such as the developments of 
inflammatory bowel disease, colonic and hepatic cancer, and atherosclerosis, problems do exist hindering the investigation on this enzyme. For example, not like other NPP members or SMases that are expressed in many cell lines, a suitable cell line that efficiently expresses the enzyme is lacking. This is presumably caused by the fact that the enzyme is down regulated or mutated in cancer cells. According to the available data from microarray studies registered in the database, it seems that only Caco-2 cells express the wild type enzyme and the expression occurs under polarized conditions [21]. The second obstacle is caused by the functional dependency of the enzyme on bile salt. Even though alk-SMase can be transfected into mammalian cells, the requirement of bile salt for its activity causes a practical problem for functional studies in vitro. We may need to modify the cell culture medium to a condition, under which the expressed enzyme can function as physiologically as possible. It is now realized that ecto-enzyme may trigger specific signal transduction pathways leading to cell proliferation and apoptosis [22, 105]. However, due to the problems mentioned above, it is still not known whether alkSMase activates specific signalling transduction pathways in addition to a common ceramide pathway. The interplay of alk-SMase and neutral ceramidase will determine the levels of ceramide, sphingosine, or even sphingosine-1-phosphate, and affect the fate of the cells differentially. The activities of alk-SMase against SM, lyso-PC and PAF may form complex cross communications among different signal transduction pathways. Generating alk-SMase knockout mice may provide important information of the enzyme regarding its functions as well as the signalling pathways.

The early occurrence and the progressive reduction of the enzyme activity associated with colon cancer raised an interesting question: whether the enzyme activity 
in the colonic mucosa or stools can be a biologic marker for diagnosis, prognosis, and routine screening. On the other hand, it is equally interesting to know whether the enzyme can be used as a chemopreventive and chemotherapeutic agent for chronic ulcerative colitis and colon cancer. These studies will further provide convincing evidence for anticancer and antiinflammatory effects of the enzyme in the colon. The importance of alk-SMase and SM digestion in the small intestine for cholesterol absorption is just emerging, and many questions are open. We may need to first investigate the impact of SM and its breakdown products in the gut on the levels and distribution of SM/cholesterol in the plasma, and the impact of inhibition of cholesterol absorption on alk-SMase expression in the small intestine. Finally, SM metabolism has been found to play important roles in infectious diseases and in immune system [106108]. Whether alk-SMase affects the functions of immunocompetent cells and the bacteria in the gut requires our attention. At least dietary sphingosine has been shown to have bactericidal effects on the epidermal layers and intestinal tract [109, 110]. With the rapid increasing knowledge of the biological effects of SM metabolites, alk-SMase, the old but now well characterized enzyme at molecular levels, can have great implications in both intestinal tract and other systems.

\section{Acknowledgement}

Dr. Åke Nilsson is acknowledged for helpful discussion and suggestions. The works cited in this review from the author's lab were supported by the grants from The Swedish Cancer Society, Albert Påhlsson's Foundation, The Swedish Research Council, and the Research Foundation of Lund University Hospital. 


\section{Reference}

[1] A. Nilsson, The presence of sphingomyelin- and ceramide-cleaving enzymes in the small intestinal tract., Biochim Biophys Acta 176 (1969) 339-347.

[2] Y. Barnholz, A. Roitman, S. Gatt, Enzymatic hydrolysis of sphingolipids. II. Hydrolysis of sphingomyelin by an enzyme from rat brain, J Biol Chem 241 (1966) 3731-3737.

[3] J.N. Kanfer, O.M. Young, D. Shapiro, R.O. Brady, The metabolism of sphingomyelin. I. Purification and properties of a sphingomyelin-cleaving enzyme from rat liver tissue, J Biol Chem 241 (1966) 1081-1084.

[4] S. Gatt, Magnesium-dependent sphingomyelinase., Biochem Biophys Res Comm 68 (1976) 235-241.

[5] P.B. Schneider, E.P. Kennedy, Sphingomyelinase in normal human spleens and in spleens from subjects with Niemann-Pick disease, J Lipid Res 8 (1967) 202-209.

[6] Y.A. Hannun, R.M. Bell, Functions of sphingolipids and sphingolipid breakdown products in cellular regulation [see comments], Science 243 (1989) 500-507.

[7] Y.A. Hannun, C.M. Linardic, Sphingolipid breakdown products: anti-proliferative and tumor- suppressor lipids, Biochim Biophys Acta 1154 (1993) 223-236.

[8] R.N. Kolesnick, F.M. Goni, A. Alonso, Compartmentalization of ceramide signaling: physical foundations and biological effects, J Cell Physiol 184 (2000) 285-300.

[9] O. Cuvillier, Sphingosine in apoptosis signaling, Biochim Biophys Acta 1585 (2002) 153-162.

[10] D. Meyer zu Heringdorf, H.M. Himmel, K.H. Jakobs, Sphingosylphosphorylcholine-biological functions and mechanisms of action, Biochim Biophys Acta 1582 (2002) 178-189.

[11] D.L. Dillehay, S.K. Webb, E.-M. Schmelz, A.H. Merrill, Dietary sphingomyelin inhibits 1,2-dimethylhydrazine-induced colon cancer in CF1 mice., J Nutr 124 (1994) 615-620.

[12] R.D. Duan, L. Nyberg, A. Nilsson, Alkaline sphingomyelinase activity in rat gastrointestinal tract: distribution and characteristics, Biochim Biophys Acta 1259 (1995) 49-55.

[13] L. Nyberg, R.D. Duan, J. Axelsson, A. Nilsson, Identification of an alkaline sphingomyelinase activity in human bile., Biochim Biophys Acta 1300 (1996) 4248.

[14] R.D. Duan, E. Hertervig, L. Nyberg, T. Hauge, B. Sternby, J. Lillienau, A. Farooqi, A. Nilsson, Distribution of alkaline sphingomyelinase activity in human beings and animals. Tissue and species differences, Dig Dis Sci 41 (1996) 18011806.

[15] R.D. Duan, Å. Nilsson, Purification of a newly identified alkaline sphingomyelinase in human bile and effects of bile salts and phosphatidylcholine on enzyme activity., Hepatology 26 (1997) 823-830.

[16] Y. Cheng, Å. Nilsson, E. Tömquist, R.D. Duan, Purification, characterization and expression of rat intestinal alkaline sphingomyelinase., J Lipid Res 43 (2002) 316-324. 
[17] R.D. Duan, Y. Cheng, G. Hansen, E. Hertervig, J.J. Liu, I. Syk, H. Sjostrom, A. Nilsson, Purification, localization, and expression of human intestinal alkaline sphingomyelinase, J Lipid Res 44 (2003) 1241-1250.

[18] R.D. Duan, T. Bergman, N. Xu, J. Wu, Y. Cheng, J. Duan, S. Nelander, C. Palmberg, A. Nilsson, Identification of Human Intestinal Alkaline Sphingomyelinase as a Novel Ecto-enzyme Related to the Nucleotide Phosphodiesterase Family, J Biol Chem 278 (2003) 38528-38536.

[19] J. Wu, Y. Cheng, C. Palmberg, T. Bergman, A. Nilsson, R.D. Duan, Cloning of alkaline sphingomyelinase from rat intestinal mucosa and adjusting of the hypothetical protein XP_221184 in GenBank, Biochim Biophys Acta 1687 (2005) 94-102.

[20] E. Hertervig, A. Nilsson, L. Nyberg, R.D. Duan, Alkaline sphingomyelinase activity is decreased in human colorectal carcinoma, Cancer 79 (1997) 448-453.

[21] J. Wu, Y. Cheng, A. Nilsson, R.D. Duan, Identification of one exon deletion of intestinal alkaline sphingomyelinase in colon cancer HT-29 cells and a differentiation-related expression of the wild-type enzyme in Caco-2 cells, Carcinogenesis 25 (2004) 1327-1333.

[22] C. Stefan, S. Jansen, M. Bollen, NPP-type ectophosphodiesterases: unity in diversity, Trends Biochem Sci 30 (2005) 542-550.

[23] J. Wu, F. Liu, A. Nilsson, R.D. Duan, Pancreatic trypsin cleaves intestinal alkaline sphingomyelinase from mucosa and enhances the sphingomyelinase activity, Am J Physiol Gastrointest Liver Physiol 287 (2004) G967-973.

[24] R.D. Duan, Y. Cheng, H.D. Tauschel, A. Nilsson, Effects of ursodeoxycholate and other bile salts on levels of rat intestinal alkaline sphingomyelinase: a potential implication in tumorigenesis, Dig Dis Sci 43 (1998) 26-32.

[25] J. Wu, G.H. Hansen, A. Nilsson, R.D. Duan, Functional studies of human intestinal alkaline sphingomyelinase by deglycosylation and mutagenesis, Biochem J 386 (2005) 153-160.

[26] H.J. Buhring, A. Streble, P. Valent, The basophil-specific ectoenzyme E-NPP3 (CD203c) as a marker for cell activation and allergy diagnosis, Int Arch Allergy Immunol 133 (2004) 317-329.

[27] N.R. Meerson, V. Bello, J.L. Delaunay, T.A. Slimane, D. Delautier, C. Lenoir, G. Trugnan, M. Maurice, Intracellular traffic of the ecto-nucleotide pyrophosphatase/phosphodiesterase NPP3 to the apical plasma membrane of MDCK and Caco-2 cells: apical targeting occurs in the absence of $\mathrm{N}$ glycosylation, J Cell Sci 113 Pt 23 (2000) 4193-4202.

[28] R. Gijsbers, H. Ceulemans, W. Stalmans, M. Bollen, Structural and catalytic similarities between nucleotide pyrophosphatases/phosphodiesterases and alkaline phosphatases, J Biol Chem 276 (2001) 1361-1368.

[29] M. Bollen, R. Gijsbers, H. Ceulemans, W. Stalmans, C. Stefan, Nucleotide pyrophosphatases/phosphodiesterases on the move, Crit Rev Biochem Mol Biol 35 (2000) 393-432.

[30] H. Sakagami, J. Aoki, Y. Natori, K. Nishikawa, Y. Kakehi, H. Arai, Biochemical and molecular characterization of a novel choline-specific glycerophosphodiester phosphodiesterase belonging to the nucleotide 
pyrophosphatase/phosphodiesterase family, J Biol Chem 280 (2005) 2308423093.

[31] S.H. Zeisel, D. Char, N.F. Sheard, Choline, phosphatidylcholine and sphingomyelin in human and bovine milk and infant formulas, J Nutr 116 (1986) 50-58.

[32] M. Blank, E.A. Cress, Z.L. Smith, F. Snyder, Meats and fish consumed in the American diet contain substantial amounts of ether-linked phospholipids., J Nutr 122 (1992) 1656-1661.

[33] W. Christie, R. Noble, G. Davies, Phospholipids in milk and dairy products., J Soc Dariy Tech 40 (1987) 10-12.

[34] L. Nyberg, Å. Nilsson, P. Lundgren, R.D. Duan, Localization and capacity of sphingomyelin digestion in the rat intestinal tract., J Nutr Biochem 8 (1997) 112118.

[35] L. Nyberg, A. Farooqi, L. Blackberg, R.D. Duan, A. Nilsson, O. Hernell, Digestion of ceramide by human milk bile salt-stimulated lipase, J Pediatr Gastroenterol Nutr 27 (1998) 560-567.

[36] L. Nyberg, Digestion and absorption of sphingomyelin from milk., Wallin \& Dalholm, Lund, 1998.

[37] P. Lundgren, A.. Nilsson, R.D. Duan, Distribution and properties of neutral ceramidase activity in rat intestinal tract., Dig Dis Sci 46 (2001) 765-772.

[38] M. Olsson, R.D. Duan, L. Ohlsson, A. Nilsson, Rat intestinal ceramidase: purification, properties, and physiological relevance, Am J Physiol Gastrointest Liver Physiol 287 (2004) G929-937.

[39] S. Mitsutake, M. Tani, N. Okino, K. Mori, S. Ichinose, A. Omori, H. Iida, T. Nakamura, M. Ito, Purification, characterization, molecular cloning, and subcellular distribution of neutral ceramidase of rat kidney, J Biol Chem 276 (2001) 26249-26259.

[40] M.S. Choi, M.A. Anderson, Z. Zhang, D.B. Zimonjic, N. Popescu, A.B. Mukherjee, Neutral ceramidase gene: role in regulating ceramide-induced apoptosis, Gene 315 (2003) 113-122.

[41] M. Kono, J.L. Dreier, J.M. Ellis, M.L. Allende, D.N. Kalkofen, K.M. Sanders, J. Bielawski, A. Bielawska, Y.A. Hannun, R.L. Proia, Neutral ceramidase encoded by the Asah2 gene is essential for the intestinal degradation of sphingolipids, $\mathrm{J}$ Biol Chem 281 (2006) 7324-7331.

[42] A. Nilsson, Metabolism of sphingomyelin in the intestinal tract of the rat., Biochim Biophys Acta 164 (1968) 575-584.

[43] N. Garmy, N. Taieb, N. Yahi, J. Fantini, Apical uptake and transepithelial transport of sphingosine monomers through intact human intestinal epithelial cells: physicochemical and molecular modeling studies, Arch Biochem Biophys 440 (2005) 91-100.

[44] J.J. Liu, Å. Nilsson, R.-D. Duan, Effects of phospholipids on sphingomyelin hydrolysis induced by intestinal alkaline sphingomyelinase: an invitro study., J Nutr Biochem 11 (2000) 192-197.

[45] J.-J. Liu, Å. Nilsson, R.-D. Duan, In vitro effects of fat, fatty acids, and cholesterol on sphingomyelin hydrolysis induced by rat intestinal alkaline sphingomyelinase, Lipids 37 (2002) 469-474. 
[46] L. Nyberg, R.D. Duan, Å. Nilsson, A mutual inhibitory effect on absorption of sphingomyelin and cholesterol., J Nutr Biochem 11 (2000) 244-249.

[47] Y. Barenholz, T.E. Thompson, Sphingomyelins in bilayers and biological membranes, Biochim Biophys Acta 604 (1980) 129-158.

[48] L.A. van Meeteren, P. Ruurs, E. Christodoulou, J.W. Goding, H. Takakusa, K. Kikuchi, A. Perrakis, T. Nagano, W.H. Moolenaar, Inhibition of autotaxin by lysophosphatidic acid and sphingosine 1-phosphate, J Biol Chem 280 (2005) 21155-21161.

[49] R.D. Duan, Hydrolysis of sphingomyelin in the gut and clinical implications in colorectal tumorigenesis and other gastrointestinal diseases., Scand J Gastroenterol 33 (1998) 673-683.

[50] E. Schmelz, A. Bushnev, D.L. Dillehan, M. Sullards, D. Liotta, A.H. Merrill, Ceramide-beta-D-glucuronide: synthesis, digestion, and suppression of early markers of colon carcinogenesis., Cancer Res 59 (1999) 5768-5772.

[51] E.M. Schmelz, A.S. Bushnev, D.L. Dillehay, D.C. Liotta, A.H. Merrill, Jr., Suppression of aberrant colonic crypt foci by synthetic sphingomyelins with saturated or unsaturated sphingoid base backbones, Nutr Cancer 28 (1997) 81-85.

[52] E. Hertervig, Nilsson.Å., J. Björk, R. Hultkrantz, R.D. Duan, Familial adenomatous polyposis is associated with a marked decrease in alkaline sphingomyelinase activity; a key factor to the unrestrained cell proliferation, $\mathrm{Br} \mathrm{J}$ Cancer 81 (1999) 232-236.

[53] L. Di Marzio, A. Di Leo, B. Cinque, D. Fanini, A. Agnifili, P. Berloco, M. Linsalata, D. Lorusso, M. Barone, C. De Simone, M.G. Cifone, Detection of alkaline sphingomyelinase activity in human stool: proposed role as a new diagnostic and prognostic marker of colorectal cancer, Cancer Epidemiol Biomarkers Prev 14 (2005) 856-862.

[54] T.A. Brasitus, P.K. Dudeja, R. Dahiya, Premalignant alterations in the lipid composition and fluidity of colonic brush border membranes of rats administered 1,2 dimethylhydrazine, J Clin Invest 77 (1986) 831-840.

[55] M. Selzner, A. Bielawska, M.A. Morse, H.A. Rudiger, D. Sindram, Y.A. Hannun, P.A. Clavien, Induction of apoptotic cell death and prevention of tumor growth by ceramide analogues in metastatic human colon cancer, Cancer Res 61 (2001) 1233-1240.

[56] M. Motouri, H. Matsuyama, J. Yamamura, M. Tanaka, S. Aoe, T. Iwanaga, H. Kawakami, Milk sphingomyelin accelerates enzymatic and morphological maturation of the intestine in artificially reared rats, J Pediatr Gastroenterol Nutr 36 (2003) 241-247.

[57] S. Chapelle, M. Gilles-Baillien, Phospholipids and cholesterol in brush border and basolateral membranes from rat intestinal mucosa, Biochim Biophys Acta 753 (1983) 269-271.

[58] D.A. Lin, J.A. Boyce, IL-4 regulates MEK expression required for lysophosphatidic acid-mediated chemokine generation by human mast cells, J Immunol 175 (2005) 5430-5438.

[59] M. Yang, W.W. Zhong, N. Srivastava, A. Slavin, J. Yang, T. Hoey, S. An, G protein-coupled lysophosphatidic acid receptors stimulate proliferation of colon 
cancer cells through the \{beta\}-catenin pathway, Proc Natl Acad Sci U S A 102 (2005) 6027-6032.

[60] B. Saatian, Y. Zhao, D. He, S.N. Georas, T. Watkins, E.W. Spannhake, V. Natarajan, Transcriptional regulation of lysophosphatidic acid-induced interleukin-8 expression and secretion by p38 MAPK and JNK in human bronchial epithelial cells, Biochem J 393 (2006) 657-668.

[61] C.C. Yun, H. Sun, D. Wang, R. Rusovici, A. Castleberry, R.A. Hall, H. Shim, LPA2 receptor mediates mitogenic signals in human colon cancer cells, Am J Physiol Cell Physiol 289 (2005) C2-11.

[62] D. Shida, J. Kitayama, H. Yamaguchi, Y. Okaji, N.H. Tsuno, T. Watanabe, Y. Takuwa, H. Nagawa, Lysophosphatidic acid (LPA) enhances the metastatic potential of human colon carcinoma DLD1 cells through LPA1, Cancer Res 63 (2003) 1706-1711.

[63] S. Jansen, C. Stefan, J.W. Creemers, E. Waelkens, A. Van Eynde, W. Stalmans, M. Bollen, Proteolytic maturation and activation of autotaxin (NPP2), a secreted metastasis-enhancing lysophospholipase D, J Cell Sci 118 (2005) 3081-3089.

[64] U. Sjöqvist, E. Hertervig, A. Nilsson, R.D. Duan, A. Ost, B. Tribukait, R. Lofberg, Chronic colitis is associated with a reduction of mucosal alkaline sphingomyelinase activity, Inflamm Bowel Dis 8 (2002) 258-263.

[65] J. Wu, A. Nilsson, B.A. Jonsson, H. Stenstad, W. Agace, Y. Cheng, R.D. Duan, Intestinal alkaline sphingomyelinase hydrolyses and inactivates platelet-activating factor by a phospholipase C activity, Biochem J 394 (2006) 299-308.

[66] T. Izumi, T. Shimizu, Platelet-activating factor receptor: gene expression and signal transduction, Biochim Biophys Acta 1259 (1995) 317-333.

[67] H. Wang, S. Chakrabarty, Platelet-activating factor activates mitogen-activated protein kinases, inhibits proliferation, induces differentiation and suppresses the malignant phenotype of human colon carcinoma cells, Oncogene 22 (2003) 21862191.

[68] Y. Denizot, A. Gainant, L. Guglielmi, S. Bouvier, P. Cubertafond, M. Mathonnet, Tissue concentrations of platelet-activating factor in colorectal carcinoma: inverse relationships with Dukes' stage of patients, Oncogene 22 (2003) 7222-7224.

[69] J.L. Wallace, G. Steel, B.J. Whittle, V. Lagente, B. Vargaftig, Evidence for platelet-activating factor as a mediator of endotoxin-induced gastrointestinal damage in the rat. Effects of three platelet-activating factor antagonists, Gastroenterology 93 (1987) 765-773.

[70] L. Ferraris, F. Karmeli, R. Eliakim, J. Klein, C. Fiocchi, D. Rachmilewitz, Intestinal epithelial cells contribute to the enhanced generation of platelet activating factor in ulcerative colitis, Gut 34 (1993) 665-668.

[71] M. Hocke, L. Richter, H. Bosseckert, K. Eitner, Platelet activating factor in stool from patients with ulcerative colitis and Crohn's disease, Hepatogastroenterology 46 (1999) 2333-2337.

[72] M.D. Amer, E. Hedlund, J. Rochester, M.S. Caplan, Platelet-activating factor concentration in the stool of human newborns: effects of enteral feeding and neonatal necrotizing enterocolitis, Biol Neonate 85 (2004) 159-166. 
[73] H. Sawai, N. Domae, N. Nagan, Y.A. Hannun, Function of the cloned putative neutral sphingomyelinase as lyso-platelet activating factor-phospholipase C, J Biol Chem 274 (1999) 38131-38139.

[74] R.D. Duan, J. Wu, E. Hertervig, Y. Cheng, A. Nilsson, Multiple forms of mutant alkaline sphingomyelinase identified in human liver biopsy samples and HepG2 cells., Gut 54 (2005) A215.

[75] H. Chen, E. Born, S.N. Mathur, F.C. Johlin, Jr., F.J. Field, Sphingomyelin content of intestinal cell membranes regulates cholesterol absorption. Evidence for pancreatic and intestinal cell sphingomyelinase activity, Biochem J 286 (1992) 771-777.

[76] F. Vrtovsnik, E.H. el Yandouzi, C. Le Grimellec, G. Friedlander, Sphingomyelin and cholesterol modulate sodium coupled uptakes in proximal tubular cells, Kidney Int 41 (1992) 983-991.

[77] E. Hertervig, A. Nilsson, Y. Cheng, R.D. Duan, Purified intestinal alkaline sphingomyelinase inhibits proliferation without inducing apoptosis in HT-29 colon carcinoma cells, J Cancer Res Clin Oncol 129 (2003) 577-582.

[78] S.K. Noh, S.I. Koo, Egg sphingomyelin lowers the lymphatic absorption of cholesterol and alpha-tocopherol in rats, J Nutr 133 (2003) 3571-3576.

[79] S.K. Noh, S.I. Koo, Milk sphingomyelin is more effective than egg sphingomyelin in inhibiting intestinal absorption of cholesterol and fat in rats, $\mathrm{J}$ Nutr 134 (2004) 2611-2616.

[80] E.R. Eckhardt, D.Q. Wang, J.M. Donovan, M.C. Carey, Dietary sphingomyelin suppresses intestinal cholesterol absorption by decreasing thermodynamic activity of cholesterol monomers, Gastroenterology 122 (2002) 948-956.

[81] N. Garmy, N. Taieb, N. Yahi, J. Fantini, Interaction of cholesterol with sphingosine: physicochemical characterization and impact on intestinal absorption, J Lipid Res 46 (2005) 36-45.

[82] S.W. Altmann, H.R. Davis, Jr., L.J. Zhu, X. Yao, L.M. Hoos, G. Tetzloff, S.P. Iyer, M. Maguire, A. Golovko, M. Zeng, L. Wang, N. Murgolo, M.P. Graziano, Niemann-Pick C1 Like 1 protein is critical for intestinal cholesterol absorption, Science 303 (2004) 1201-1204.

[83] A.J. Benade, J.E. Fincham, C.M. Smuts, M.T. Tung, D. Chalton, M. Kruger, M.J. Weight, A.K. Daubitzer, H.Y. Tichelaar, Plasma low density lipoprotein composition in relation to atherosclerosis in nutritionally defined Vervet monkeys, Atherosclerosis 74 (1988) 157-168.

[84] S.L. Schissel, J. Tweedie-Hardman, J.H. Rapp, G. Graham, K.J. Williams, I. Tabas, Rabbit aorta and human atherosclerotic lesions hydrolyze the sphingomyelin of retained low-density lipoprotein. Proposed role for arterial-wall sphingomyelinase in subendothelial retention and aggregation of atherogenic lipoproteins, J Clin Invest 98 (1996) 1455-1464.

[85] E.B. Smith, Intimal and medial lipids in human aortas, Lancet April (1960) 799803.

[86] O.W. Portman, M. Alexander, Metabolism of sphingolipids by normal and atherosclerotic aorta of squirrel monkeys, J Lipid Res 11 (1970) 23-30.

[87] S. Eisenberg, Y. Stein, O. Stein, Phospholipase in arterial tissue. III. Phosphatide acyl-hydrolase, lysophosphatide acyl-hydrolase and sphingomyelin cholin 
phosphohydrolase in rat and rabbit aorta in different age groups., Biochim Biophys Acta 176 (1969) 557-569.

[88] S. Eisenberg, Y. Stein, O. Stein, Phospholipases in arterial tissue. IV. The role of phosphatide acyl hydrolase, lysophosphatide acyl hydrolase, and sphingomyelin choline phosphohydrolase in the regulation of phospholipid composition in the normal human aorta with age., J Clin Invest 48 (1969) 2320-2329.

[89] J.D. Bagdade, P.V. Subbaiah, Abnormal high-density lipoprotein composition in women with insulin-dependent diabetes, J Lab Clin Med 113 (1989) 235-240.

[90] J.D. Bagdade, W.E. Buchanan, T. Kuusi, M.R. Taskinen, Persistent abnormalities in lipoprotein composition in noninsulin-dependent diabetes after intensive insulin therapy, Arteriosclerosis 10 (1990) 232-239.

[91] C. Noel, Y.L. Marcel, J. Davignon, Plasma phospholipids in the different types of primary hyperlipoproteinemia, J Lab Clin Med 79 (1972) 611-621.

[92] A. Schlitt, M.R. Hojjati, H. von Gizycki, K.J. Lackner, S. Blankenberg, B. Schwaab, J. Meyer, H.J. Rupprecht, X.C. Jiang, Serum sphingomyelin levels are related to the clearance of postprandial remnant-like particles, J Lipid Res 46 (2005) 196-200.

[93] X.P. Yang, D. Yan, C. Qiao, R.J. Liu, J.G. Chen, J. Li, M. Schneider, L. Lagrost, X. Xiao, X.C. Jiang, Increased atherosclerotic lesions in apoE mice with plasma phospholipid transfer protein overexpression, Arterioscler Thromb Vasc Biol 23 (2003) 1601-1607.

[94] D. Yan, M. Navab, C. Bruce, A.M. Fogelman, X.C. Jiang, PLTP deficiency improves the anti-inflammatory properties of HDL and reduces the ability of LDL to induce monocyte chemotactic activity, J Lipid Res 45 (2004) 1852-1858.

[95] Z. Li, M.J. Basterr, T.K. Hailemariam, M.R. Hojjati, S. Lu, J. Liu, R. Liu, H. Zhou, X.C. Jiang, The effect of dietary sphingolipids on plasma sphingomyelin metabolism and atherosclerosis, Biochim Biophys Acta 1735 (2005) 130-134.

[96] Y. Cheng, L. Ohlsson, R.D. Duan, Psyllium and fat in diets differentially affect the activities and expressions of colonic sphingomyelinases and caspase in mice, Br J Nutr 91 (2004) 715-723.

[97] A.-M. Pajari, P. Häkkänen, R.D. Duan, M. Mutanen, Role of red meat and arachidonic acid in protein kinase $\mathrm{C}$ activation in rat colonic musosa., Nutr \& Cancer 32 (1998) 86-94.

[98] L. Yang, M. Mutanen, Y. Cheng, R.D. Duan, Effects of fat, beef and fiber in diets on activities of sphingomyelinase, ceramidase and caspase-3 in rat colonic mucosa, Med Princ Pract 11 (2002) 150-156.

[99] Y. Cheng, H.-T. Tauschel, Å. Nilsson, R.D. Duan, Administration of ursodeoxycholic acid increases the activities of alkaline sphingomyelinase and caspase-3 in rat colon., Scand J Gastroenterol 34 (1999) 915-920.

[100] F. Liu, Y. Cheng, J. Wu, H.-D. Tauschel, R.D. Duan, Ursodeoxycholic acid differentially affects three types of sphingomyelinase in human colon cancer Caco-2 cells., Cancer Lett 235 (2006) 141-146.

[101] G.C. Kaiser, F. Yan, D.B. Polk, Mesalamine blocks tumor necrosis factor growth inhibition and nuclear factor kappaB activation in mouse colonocytes, Gastroenterology 116 (1999) 602-609. 
[102] R.D. Duan, Potential link between sphingomyelin metabolism and colonic tumorigenesis., in: W. Scheppach, M. Scheurlen (Eds.), Exogenous factors in colonic carcinogenesis, Kluwer Academic Publishers, Lancaster, UK., 2003, pp. 142-153.

[103] D.L. Earnest, H. Holubec, R.K. Wali, C.S. Jolley, M. Bissonette, A.K. Bhattacharyya, H. Roy, S. Khare, T.A. Brasitus, Chemoprevention of azoxymethane-induced colonic carcinogenesis by supplemental dietary ursodeoxycholic acid, Cancer Res 54 (1994) 5071-5074.

[104] D.T. Rubin, B.A. Lashner, Will a 5-ASA a day keep the cancer (and dysplasia) away?, Am J Gastroenterol 100 (2005) 1354-1356.

[105] J.W. Goding, Ecto-enzymes: physiology meets pathology, J Leukoc Biol 67 (2000) 285-311.

[106] L.R. Ballou, S.J. Laulederkind, E.F. Rosloniec, R. Raghow, Ceramide signalling and the immune response, Biochim Biophys Acta 1301 (1996) 273-287.

[107] K. Hanada, Sphingolipids in infectious diseases, Jpn J Infect Dis 58 (2005) 131148.

[108] T. Baumruker, F. Bornancin, A. Billich, The role of sphingosine and ceramide kinases in inflammatory responses, Immunol Lett 96 (2005) 175-185.

[109] D.J. Bibel, R. Aly, H.R. Shinefield, Antimicrobial activity of sphingosines, J Invest Dermatol 98 (1992) 269-273.

[110] S. Possemiers, J. Van Camp, S. Bolca, W. Verstraete, Characterization of the bactericidal effect of dietary sphingosine and its activity under intestinal conditions, Int J Food Microbiol 105 (2005) 59-70. 
Table 1. Important events in alkaline sphingomyelinase research

Time Finding Ref

1969 Identify the activity in the intestine 1

1995 Characterize the distribution and properties of the enzyme

1996 Identify alk-SMase activity in human bile 13

1997 Identify decrease of the enzyme in human colon cancer 20

1998 Purify the enzyme in human bile 15

2002 Identify decrease of the enzyme in inflammatory bowel diseases 64

$2002 \quad$ Purify rat intestinal alk-SMase. 16

2003 Purify and clone human alk-SMase, find it to be a NPP member. 18

$2003 \quad$ Identify inhibitory effect of the enzyme on colon cancer cells. 77

$2004 \quad$ Identify the mutation associated with human colon cancer. $\quad 21$

2005 Clone the enzyme from rat intestinal tissue 19

2005 Identify a novel activity against PAF 65 


\section{Figure legends.}

Fig. 1. The schematic structure of intestinal alk-SMase. The five translated exons of alkSMase gene are shown in different colours. The positions of the important amino acid residues are indicated. The domain from A5 and A22 is a signal peptide and that from P441 to V437 is a signal anchor. The domain between T73 and H79 is a predicted catalytic core. The $5 \mathrm{~N}$-glycosylation sites are indicated at the top and the residues forming two metal coordinating sites are indicated in red and blue, respectively.

Fig. 2. Digestion of dietary SM in human after a meal. Human has two sources of alkSMase: one in the bile and the other in the intestinal mucosa. After a Western meal, the released CCK will stimulate the contraction of gallbladder and deliver bile alk-SMase together with bile salts in the intestine. Meanwhile CCK will increase the secretion of pancreatic trypsin, which together with bile salt will dissociate both intestinal alk-SMase and neutral ceramidase from the mucosa into the lumen. SM will be sequentially digested by alk-SMase and neutral ceramidase in the presence of bile salt. The produced sphingosine is absorbed in the epithelial cells and converted either to fatty acid or to ceramide.

Fig. 3. Anticancer effects of alk-SMase. Alk-SMase exerts its anticancer effects by three arms: hydrolysing SM to form ceramide, hydrolysing lyso-PC to reduce LPA production and hydrolysing PAF to inhibit its activity. The key molecules that mediate the alkSMase effects are listed in each box. 
Fig. 4. Mutation of alk-SMase found in colon and liver cancer. The wild type mRNA contains 5 translated exons as indicated in different colours. The variants of the mRNA found in colon and liver tumours due to alternative splicing are shown in corresponding colours of the exons. The additional insert and the tag are indicated. 
Fig. 1

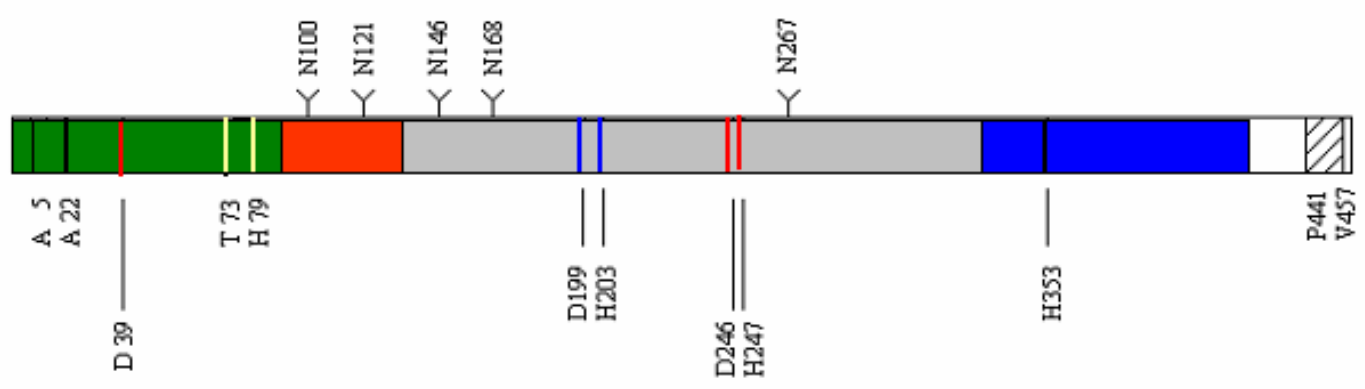

Exon 1

2

3

4

5 
Fig. 2

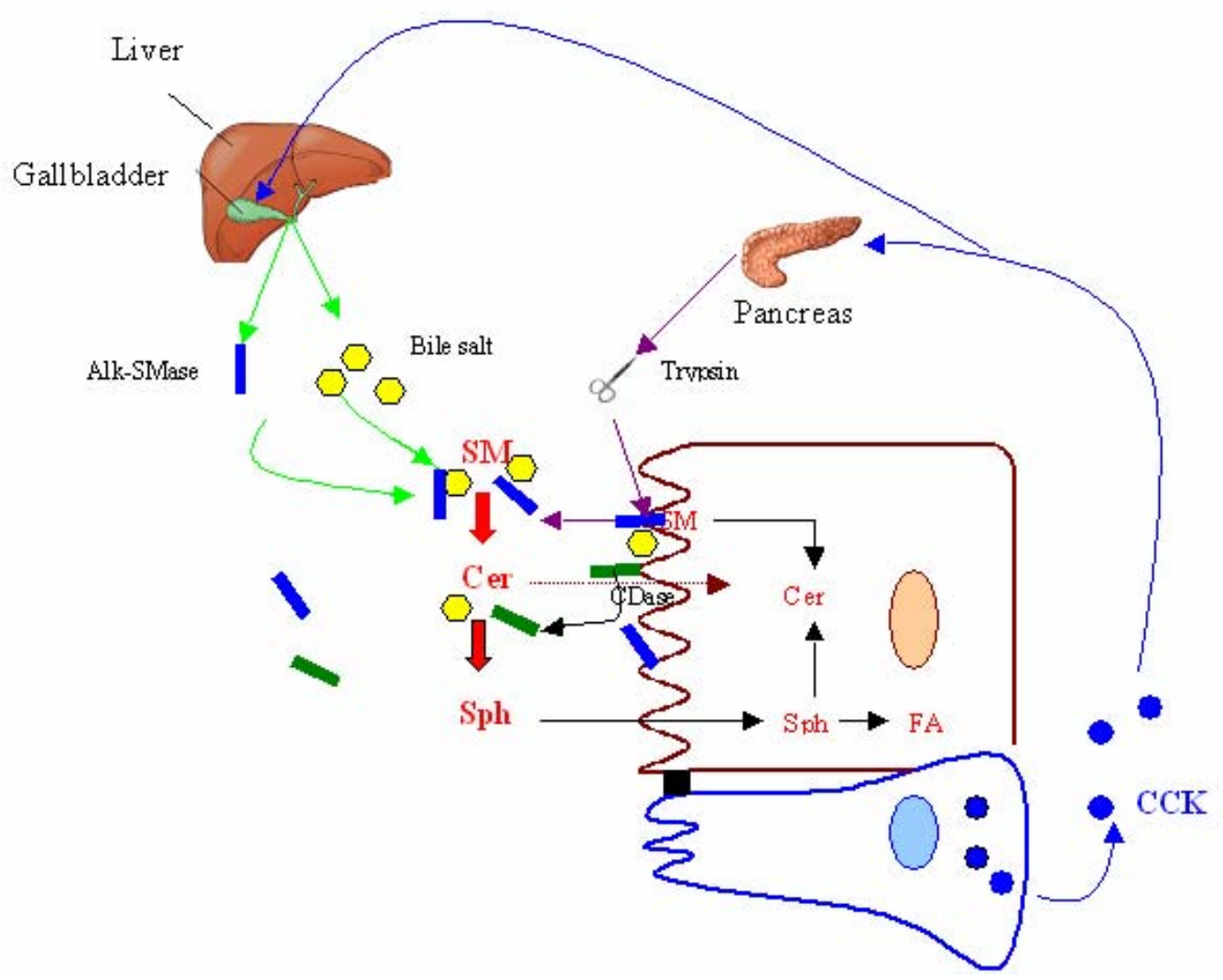


Fig. 3.

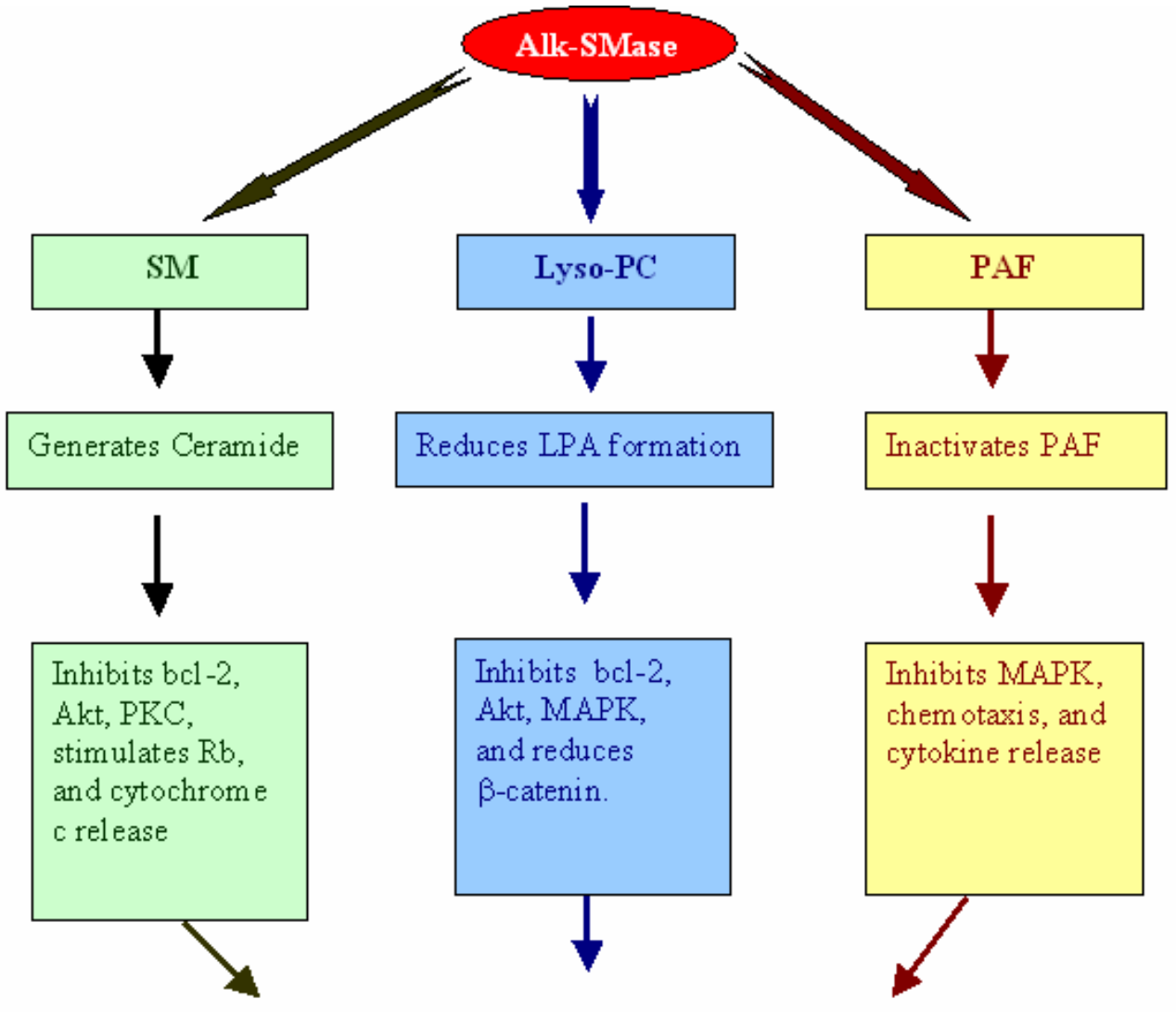

Anti prolifer ative, anti-metastasis, and pro-ap op totic effects 
Fig. 4.

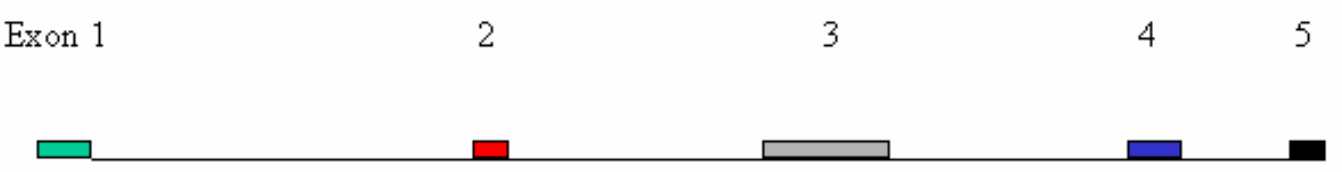

Wild type

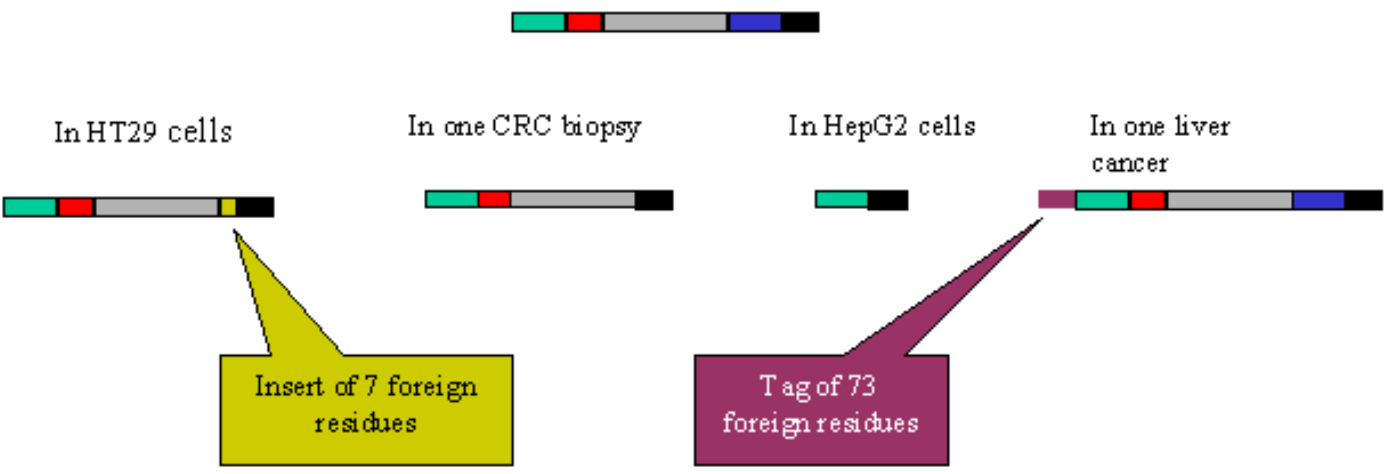

